\title{
The Factors That Affect Improvement of Neurogenic Bladder by Severe Lumbar Disc Herniation in Operation
}

\author{
Joon Bok Jeon ${ }^{1}$, Seung Hwan Yoon ${ }^{1}$, Do Keun Kim¹, Ji-Yong Kim² \\ ${ }^{1}$ Department of Neurosurgery, Inha University School of Medicine, Incheon, \\ ${ }^{2}$ Department of Neurosurgery, Naeun Hospital, Incheon, Korea
}

\begin{abstract}
Objective: This study analyzed retrospectively the bladder function of patients after early surgery for cauda equina syndrome (CES) performed within 24 or 48 hours, or after 48 hours of the onset of autonomic symptoms.

Methods: We retrospectively reviewed the clinical data of 31 patients after decompression surgery for lumbar disc herniation (LDH) who had been diagnosed with CES between January 2001 and December 2014 at Inha University Hospital. The following factors were assessed to evaluate the influence of time to surgery: bladder function, rectal incontinence, sexual dysfunction, LDH level, and degree of spinal canal compression.

Results: After decompression, the outcome group was categorized into normal bladder function and abnormal bladder function. The patients operated on within 48 hours showed an improved postoperative outcome. Among 16 patients operated on within 48 hours, $13(81 \%)$ recovered normal bladder function. In contrast, among 15 patients with decompression after 48 hours, $6(40 \%)$ recovered normal bladder function. Among 21 patients with mild bladder dysfunction at admission, $16(76 \%)$ recovered normal bladder function after decompression.

Conclusion: Our study suggests that patients who have decompression surgery within 48 hours of the onset of bladder dysfunction, improve their chances of recovering bladder function than those who have a late operation ( $>48$ hours). Also, patients with mild bladder dysfunction are more likely to recover bladder function after decompression, than patients with severe bladder dysfunction.
\end{abstract}

Key Words: Polyradiculopathy $\cdot$ Treatment outcome $\cdot$ Intervertebral disc displacement $\cdot$ Neurogenic urinary bladder $\cdot$ Spinal cord compression

\section{INTRODUCTION}

The classic presentation of cauda equine syndrome (CES) is a middle-aged adult complaining of bilateral sciatica, bilateral lower extremity weakness, particularly below the knees, saddle anesthesia, and urinary or rectal incontinence ${ }^{8)}$. CES can be caused by a lumbar disc herniation (LDH). The most common spinal segments involved are L4-L5 and L5-S1. Other etiologies include spinal stenosis, spinal tumors, hematomas, fractures, and infections ${ }^{2}$. CES is a rare but devastating medical condition, requiring urgent surgery to improve neurological symptoms. CES progresses to paraplegia and permanent urinary or rectal incontinence, hence, CES is considered the absolute indication for lumbar disc surgery ${ }^{8,9}$. Some resear-

- Received: July 12, 2016 • Revised: August 29, 2016

- Accepted: September 7, 2016

Corresponding Author: Seung Hwan Yoon

Department of Neurosurgery, Inha University School of Medicine,

366 Seohae-daero, Jung-gu, Incheon 22332, Korea

Tel: +82-32-890-2619, Fax: +82-32-890-2619

E-mail: nsyoon@gmail.com

$\otimes$ This is an open access article distributed under the terms of the Creative Commons Attribution Non-Commercial License (http://creativecommons.org/licenses/by-nc/4.0/) which permits unrestricted non-commercial use, distribution, and reproduction in any medium, provided the original work is properly cited. chers have compared the urgency of CES surgery to that of evacuating an epidural hematoma after severe head trauma ${ }^{6}$.

There is considerable debate regarding the appropriate timing of surgery for $\mathrm{CES}^{10)}$. A meta-analysis by Ahn et al. ${ }^{1)}$ recommended operating within 48 hours of the onset of symptoms, due to the significant improvement in both sensory and motor disability as well as urinary function. It is generally accepted that surgical treatment of CES should be performed as soon as possible, specifically, within 48 hours of the onset of symptoms.

We analyzed retrospectively whether early decompression surgery for CES, within 24 or 48 hours, or after 48 hours of the onset of autonomic symptoms influenced postoperative bladder function. In addition, several other factors that determine the success of the surgery outcome were analyzed to permit accurate treatment decisions. These factors included rectal incontinence, sexual dysfunction, LDH level and degree of spinal canal compression.

\section{MATERIALS AND METHODS}

We retrospectively reviewed the clinical data of 31 patients who had decompressive surgery for $\mathrm{LDH}$ and were diagnosed with CES between January 2001 and December 2014 at Inha 
University Hospital. All patients complained of urinary dysfunction by acute or chronic LDH but other symptoms due to $\mathrm{LDH}$, such as motor weakness, radiating pain, rectal dysfunction, and sexual dysfunction were or were not presented in enrolled patients. Acute and chronic LDH were defined that any symptom except for urinary dysfunction such as radiating pain, weakness was presented in patients within less than 6 weeks from onset of urinary dysfunction or more than 6 weeks from urinary dysfunction. An LDH below L2 level was included in this study and the improvement of initial symptoms after surgery were evaluated over a follow-up between 12-96 months. After surgery, patients had been classified into a normal bladder function group and an abnormal bladder function group, according to their bladder function improvement. Abnormal bladder function was defined as requiring clean intermittent catheterization because of urinary difficulty. We also assessed whether the time to operation from the onset of autonomic symptom, rectal dysfunction, sexual dysfunction (male only), main level of disc herniation and degree of spinal canal compression correlated with surgical outcome. The data used to evaluate the surgical outcomes were collected from clinical charts, out-patient department surveys and interview call at December 2015. We defined the time to operation as the duration between the onset of urinary dysfunction and decompression surgery. Three categories were identified: less than 24 hours, 24-48 hours, and more than 48 hours. The symptoms were defined as follows: rectal dysfunction, as having difficulty defecating with decreased perianal sense; sexual dysfunction, as having difficulty achieving erection (in males only); level of herniated disc, as the level at which most severe spinal canal compression occurred; and degree of spinal canal compression, as the ratio of herniated disc anteroposterior (AP) diameter of the spinal canal to AP diameter of a normal spinal canal (Fig. 1). The severity of urinary dysfunction at admission was categorized as mild (did not require Foley catheterization) and severe (required Foley catheterization).

The data were statistically analyzed by IBM SPSS Statistics ver. 22.0 (IBM Co., Armonk, NY, USA) and the odds ratio determined using Pearson chi-square test.

\section{RESULTS}

A total of 31 patients (mean age, 50.7 years; median, 51 years; range, 19-83 years), comprising 26 male and 5 female patients were assessed during a 12- to 96 -month follow-up.

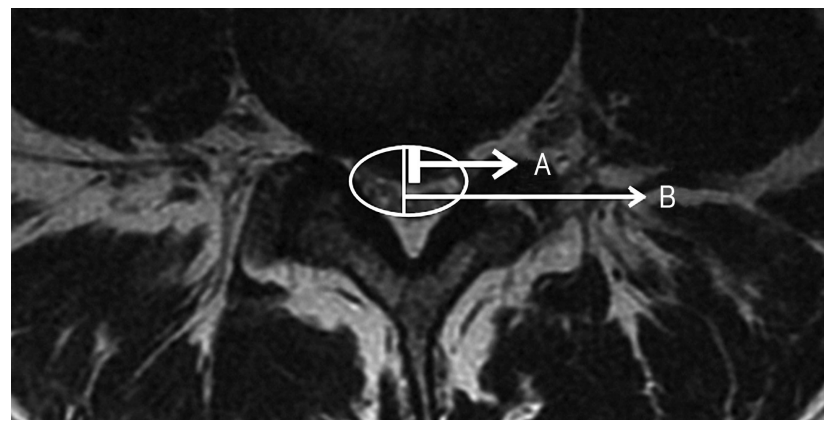

Fig. 1. A is the anteroposterior diameter of a herniated disc at a level defined as mainly compressed. B is the anteroposterior diameter of the spinal canal at a level defined as mainly compressed. The degree of spinal canal compression $(\%)=\mathrm{A} / \mathrm{B} \times 100$.

Table 1. Information of enrolled patients

\begin{tabular}{|c|c|c|c|c|}
\hline Variable & Less than 24 hours & 24-48 Hours & More than 48 hours & Total \\
\hline \multicolumn{5}{|c|}{ Initial severity of bladder symptom } \\
\hline Mild & 5 & 5 & 11 & 21 \\
\hline Severe & 3 & 3 & 4 & 10 \\
\hline \multicolumn{5}{|l|}{ Rectal dysfunction } \\
\hline Yes & 6 & 5 & 7 & 18 \\
\hline No & 2 & 3 & 8 & 13 \\
\hline \multicolumn{5}{|c|}{ Sexual dysfunction (male only) } \\
\hline Yes & 1 & 3 & 4 & 8 \\
\hline No & 6 & 4 & 8 & 18 \\
\hline \multicolumn{5}{|c|}{ Main level of disc herniation } \\
\hline L23 & 0 & 0 & 2 & 2 \\
\hline L34 & 3 & 2 & 4 & 9 \\
\hline L45 & 3 & 4 & 5 & 12 \\
\hline L5S1 & 2 & 2 & 4 & 8 \\
\hline \multicolumn{5}{|l|}{ Follow-up period } \\
\hline Under 3 years & 4 & 8 & 2 & 14 \\
\hline $3-6$ years & 2 & 5 & 3 & 10 \\
\hline $6-9$ years & 2 & 2 & 3 & 7 \\
\hline Total & 8 & 8 & 15 & 31 \\
\hline
\end{tabular}


Fourteen patients were evaluated for outcome on within 3 years after operation. 10 patients were evaluated on 3-6 years after operation and 7 patients were evaluated on $6^{-9}$ years after operation. Details for patients are in Table 1. A total of 8 patients (22.6\%) were operated on within 24 hours. After decompression, 7 of these patients had normal bladder function and one had abnormal bladder function. Of the 23 patients with decompression after 24 hours, 12 had a normal bladder function outcome and 11 had an abnormal bladder function outcome (Table 2). The proportion of patients with normal bladder function outcome was higher when decompression was performed within 24 hours compared to after 24 hours but there was not statistically significant difference (Table 2). Of the 16 patients operated on within 48 hours, 13 and 3 patients had normal and abnormal bladder function outcome, respectively. Of the 15 patients with decompression after 48 hours, 6 and 9 had normal and abnormal bladder function outcome, respectively. Patients operated on within 48 hours compared to after 48 hours, had a significantly better outcome after decompression (Table 2). The group operated on within 48 hours were 6.5 times more likely to have normal bladder function after decompression compared to the group with decompre- ssion after 48 hours (odds ratio, 6.5; 95\% confidence interval, 1.27 to 33.03; Fisher exact test 2-sided, $p=0.029$ ). There were no complications, such as dural tears and infection, during the perioperative period.

The degree of spinal canal compression, which ranged between $10 \%$ and $80 \%$, was categorized into less than 50\% (16 patients) and more than 50\% (15 patients). After decompression, a respective 11 and 5 patients had normal and abnormal bladder function for patients with less than 50\% compression. For patients with more than $50 \%$ compression, 8 and 7 patients had normal and abnormal bladder function, respectively, after decompression. The proportion of patients with normal bladder function outcome was higher in patients with less than $50 \%$ compression (68.7\%) compared to more than $50 \%(53.3 \%)$. But there was no statistically significant difference $(\mathrm{p}=0.371)$ in the degree of spinal canal compression to the surgical outcome (Table 3).

There were 18 and 13 patients with and without rectal dysfunction, respectively. After decompression, respective 11 and 7 patients had normal and abnormal bladder function for patients with rectal dysfunction. For patients without rectal dysfunction, 8 and 5 patients had normal and abnormal bladder

Table 2. Timing of operation and outcome of bladder function after decompression

\begin{tabular}{ccccc}
\hline \hline \multirow{2}{*}{ Bladder function } & \multicolumn{3}{c}{ Timing of operation } & \multirow{2}{*}{ p-value } \\
\cline { 2 - 4 } & Less than 24 hours & $24-48$ Hours & More than 48 hours & 0.029 \\
Normal bladder & 7 & 6 & 6 & 9 \\
Abnormal bladder & 1 & 2 & 9 & \\
\hline " $\mathrm{p}<0.05$, statistically significant difference; less than 48 hours vs. more than 48 hours: Fisher exact test 2-sided.
\end{tabular}

Table 3. Other factors and outcome after decompression

\begin{tabular}{|c|c|c|c|}
\hline Variable & Normal bladder & Abnormal bladder & p-value \\
\hline Initial severity of bladder symptom & & & $0.021^{*, a}$ \\
\hline Mild & $16 / 21(76.0)$ & $5 / 21(24.0)$ & \\
\hline Severe & $3 / 10(30.0)$ & $7 / 10(70.0)$ & \\
\hline Rectal dysfunction & & & $0.981^{b}$ \\
\hline Yes & $11 / 18(61.1)$ & $7 / 18(38.9)$ & \\
\hline No & $8 / 13(61.5)$ & $5 / 13(38.5)$ & \\
\hline Sexual dysfunction (male only) & & & $0.671^{b}$ \\
\hline Yes & $6 / 8(75.0)$ & $2 / 8(25.0)$ & \\
\hline No & 12/18 (66.6) & 6/18 (33.4) & \\
\hline Main level of disc herniation & & & $0.125^{\mathrm{c}}$ \\
\hline $\mathrm{L} 23$ & $1 / 2(50.0)$ & $1 / 2(50.0)$ & \\
\hline L34 & 3/9 (33.3) & $6 / 9(66.4)$ & \\
\hline L45 & $8 / 12(66.6)$ & $4 / 12(33.4)$ & \\
\hline L5S1 & $5 / 8(62.5)$ & $3 / 8(37.5)$ & \\
\hline Degree of spinal canal compression & & & $0.379^{\mathrm{b}}$ \\
\hline Less than $50 \%$ & $11 / 16(68.7)$ & $5 / 16(31.3)$ & \\
\hline More than $50 \%$ & 8/15 (53.3) & 7/15 (46.7) & \\
\hline
\end{tabular}


function, respectively, after decompression. The proportion of patients with normal bladder function outcome was almost same in patients with rectal dysfunction (61.1\%) and without rectal dysfunction (61.5\%). There was no statistically signifi cant difference $(\mathrm{p}=0.981)$ in the rectal dysfunction to the surgical outcome. There were eight and 18 male patients with and without sexual dysfunction, respectively. After decompression, respective 6 and 2 patients had normal and abnormal bladder function for patients with sexual dysfunction. For patients without sexual dysfunction, 12 and 6 patients had normal and abnormal bladder function, respectively, after decompression. The proportion of patients with normal bladder function outcome was higher in patients with sexual dysfunction (75.0 $\%)$ compared to patients without sexual dysfunction (66.6\%). But there was no statistically significant difference $(p=0.671)$ in the sexual dysfunction to the surgical outcome. There were 11 and 20 patients with $\mathrm{L} 234 \mathrm{LDH}$ and L45S1 LDH, respectively. After decompression, respective 4 and 7 patients had normal and abnormal bladder function for patients with L234 LDH. For patients with L45S1, 13 and 7 patients had normal and abnormal bladder function, respectively, after decompression. The proportion of patients with normal bladder function outcome was higher in patients with $\mathrm{L} 45 \mathrm{~S} 1 \mathrm{LDH}(65.0 \%)$ compared to patients with $\mathrm{L} 234 \mathrm{LDH}(36.3 \%)$. But there was no statistically significant difference $(\mathrm{p}=0.125)$ in the main level of LDH to the surgical outcome (Table 3).

Of the 21 patients with mild bladder dysfunction at admission, a respective 16 and 5 had normal and abnormal bladder function after decompression. Among the 10 patients with severe bladder dysfunction at admission, 3 and 7 had normal and abnormal bladder function after decompression. The proportion of patients with normal bladder function outcome was higher about 2.5 times in patients with mild bladder dysfunction at admission (76.0\%) compared to patients with severe bladder dysfunction (30.0\%). There was a statistically significant difference $(\mathrm{p}=0.021)$ in the severity of bladder dysfunction at admission to the surgical outcome (Table 3).

Consequently decompression resulted in a significantly impro ved outcome and there is a statistically significant difference in this study for patients on operated within 48 hours and with mild bladder dysfunction at admission.

\section{DISCUSSION}

CES can occur due to $\mathrm{LDH}$, tumor, or epidural abscess, and so on ${ }^{4)}$. But CES is a rare and severe condition and is considered the absolute surgical indication ${ }^{8,9)}$.

The pathological CES process is debated but the mechanism of injury to the nerves in CES is suggested to be due to the combination of mechanical pressure, arterial ischemia, and venous congestion $^{3)}$. Therefore, decompressive surgery is essential to improve the disability of this condition. The timing of surgical intervention for CES by decompression is debatable. Ahn et al. ${ }^{1)}$ from John Hopkins University demonstrated that treating all patients with CES within 48 hours of autonomic symptoms led to significant improvement in sensory and motor deficits, and urinary and rectal function. However, Kohles et al. ${ }^{5)}$ suggested that a significant improvement in outcome was seen with decompression within 24 hours. Interestingly, one prospective study analyzed 33 patients undergoing surgery for CES due to $\mathrm{LDH}^{7}$, and found no statistical significance between timing of surgery (less than 24 hours, 24-48 hours, more than 48 hours) but a better outcome after decompression was found for patients who were continent of urine at onset of symptom than for patients who were incontinent of urine. This result supports the theory that the severity of bladder dysfunction at the onset of symptom may be a factor determining the outcome after decompression.

Our study shows that operating within 48 hours on CES patients with bladder dysfunction caused by $\mathrm{LDH}$ can results in a significantly improved postsurgical outcome (6.5 fold) compared to decompression after 48 hours. Although the proportion of patients presenting a better postsurgery when operated on within 24 hours was higher than the patients with decompression after 24 hours, it was not statistical significant difference because p-value is 0.108 . Furthermore, patients with mild bladder dysfunction at admission had an improved outcome after operation compared with patients with severe bladder dysfunction. But our study did not show that other factors, level of $\mathrm{LDH}$, degree of spinal canal compression, sexual dysfunction, rectal dysfunction, are related to outcome. There was a little improved outcome after operation in patients with sexual dysfunction, lower level of LDH (L45S1), and less than $50 \%$ spinal canal compression but there was no statistically significant difference $(p>0.05)$. And there was no difference of outcome between with and without rectal dysfunction.

There were several limitations to this study. This study is small, total 31 cases. It was a retrospective study; recent information regarding the surgical outcome was obtained by phone interview but most data were collected from medical records, including time to operation and severity of symptoms. In addition, because most patients with urinary dysfunction were operated on immediately or one day after arrival, the evaluation of bladder function was assessed by objective tests, such as uroflowmetry and postvoid residual urine scans were only performed in some patients. Furthermore, and in contrast to the objective evaluation, the outcome of bladder dysfunction after surgery was evaluated subjectively through a statement and survey. Follow-up period also was various from 12 months to 96 months. Longer follow-up period can result in confused outcome after operation because of other disease affecting the outcome such as benign prostatic hyperplasia, urethral stricture. If follow-up period for patients is equal, more reliable result can be showed.

\section{CONCLUSION}

Our study suggests that decompression surgery should be performed for patients with a neurogenic bladder within 48 hours from the onset of bladder dysfunction to improve bladder dys- 
function after decompression. In addition, patients with mild bladder dysfunction at admission showed a significantly improved outcome after decompression.

\section{CONFLCT OF INTEREST}

No potential conflict of interest relevant to this article was reported.

\section{REFERENCES}

1. Ahn UM, Ahn NU, Buchowski JM, Garrett ES, Sieber AN, Kostuik JP: Cauda equina syndrome secondary to lumbar disc herniation: a meta-analysis of surgical outcomes. Spine (Phila Pa 1976) 25:1515-1522, 2000

2. Gardner A, Gardner E, Morley T: Cauda equina syndrome: a review of the current clinical and medico-legal position. Eur Spine J 20:690-697, 2011

3. Gleave JR, MacFarlane R: Prognosis for recovery of bladder function following lumbar central disc prolapse. Br J Neurosurg 4:205-209, 1990
4. Ju JH, Kim HW, Jung CK, Ha HG: Sudden onset of cauda equina syndrome resulting from posterior migration of lumbar herniated disc without significant previous neurological signs. Korean J Spine 9:281-284, 2012

5. Kohles SS, Kohles DA, Karp AP, Erlich VM, Polissar NL: Timedependent surgical outcomes following cauda equina syndrome diagnosis: comments on a meta-analysis. Spine (Phila Pa 1976) 29:1281-1287, 2004

6. O'Laoire SA, Crockard HA, Thomas DG: Prognosis for sphincter recovery after operation for cauda equina compression owing to lumbar disc prolapse. Br Med J (Clin Res Ed) 282:1852-1854, 1981

7. Qureshi A, Sell P: Cauda equina syndrome treated by surgical decompression: the influence of timing on surgical outcome. Eur Spine J 16:2143-2151, 2007

8. Shapiro S: Cauda equina syndrome secondary to lumbar disc herniation. Neurosurgery 32:743-746, 1993

9. Shephard RH: Diagnosis and prognosis of cauda equina syndrome produced by protrusion of lumbar disk. Br Med J 2:1434 1439, 1959

10. Todd NV: Cauda equina syndrome: the timing of surgery probably does influence outcome. Br J Neurosurg 19:301-306, 2005 